\title{
Tyrosol and its metabolites as antioxidative and anti-inflammatory molecules in human endothelial cellst
}

Francisco J. G. Muriana *a, Sergio Montserrat-de la Paz ${ }^{a}$, Ricardo Lucas ${ }^{b}$, Beatriz Bermudez ${ }^{\text {c }}$, Sara Jaramillo ${ }^{\mathrm{d}}$, Juan C. Morales , Rocio Abia ${ }^{\mathrm{a}}$ and Sergio Lopez $*^{\mathrm{a}}$

${ }^{a}$ Laboratory of Cellular and Molecular Nutrition, Instituto de la Grasa (CSIC), Seville, Spain. E-mail: serglom@ig.csic.es; muriana@ig.csic.es; Fax: +34954616790; Tel: $+34954611550$

${ }^{\mathrm{b}}$ Department of Biochemistry and Molecular Pharmacology, Instituto de Parasitologia y Biomedicina (CSIC), Granada, Spain ${ }^{c}$ Department of Cell Biology, School of Biology (University of Seville), 41012 Seville, Spain

${ }^{\mathrm{d}}$ Phytochemicals and Food Quality Group, Instituto de la Grasa (CSIC), Seville, Spain

Received 28th April 2017 , Accepted 3rd July 2017

First published on 4th July 2017

Tyrosol (Tyr) is a phenolic compound found in virgin olive oil. After ingestion, Tyr undergoes extensive first pass intestinal/hepatic metabolism. However, knowledge about the biological effects of Tyr metabolites is scarce. We chemically synthesized Tyr glucuronate (Tyr-GLU) and sulphate (Tyr-SUL) metabolites and explored their properties against oxidative stress and inflammation in TNF- $\alpha$-treated human umbilical vein endothelial cells (hECs). Tyr and Tyr-SUL prevented the rise of reactive oxygen species, the depletion of glutathione, and the down-regulation of glutathione peroxidase 1 , glutamate-cysteine ligase catalytic subunit, and heme oxygenase-1 genes. Tyr-SUL and to a lower extent Tyr and Tyr-GLU prevented the phosphorylation of NF- $\kappa$ B signaling proteins. Tyr-GLU and Tyr-SUL also prevented the over-expression of adhesion molecules at gene, protein, and secretory levels, and the adhesion (Tyr-SUL > Tyr-GLU) of human monocytes to hECs. In vivo, Tyr, and most notably Tyr-SUL in a dose-dependent manner, ameliorated plantar and ear edemas in mice models of acute and chronic inflammation. This study demonstrates the antioxidant and/or antiinflammatory properties of Tyr metabolites, with Tyr-SUL being the most effective. 


\section{Introduction}

Polyphenols from virgin olive oil have been suggested to be responsible at least for part of the health benefits of virgin olive oil consumption against a range of chronic inflammatory diseases such as atherosclerosis. ${ }^{-}$Around $80-90 \%$ of the total phenol content of the virgin olive oil can be found as secoiridoids, which are represented by the dialdehydic and aldehydic forms of the aglycons: oleuropein (3,4dihydroxyphenylethanol-elenolic acid dialdehyde and 3,4-dihydroxyphenylethanolelenolic acid, respectively) and ligstroside (p-hydroxyphenylethanol-elenolic acid dialdehyde and p-hydroxyphenylethanol-elenolic acid, respectively). ${ }^{\underline{2}}$ Under gastric conditions, secoiridoids are hydrolysed, resulting in a significant increase in the amounts of free polyphenols available for absorption in a dose-dependent manner. ${ }^{2}$ Thereafter, along with 3,4-dihydroxyphenylethanol (hydroxytyrosol), phydroxyphenylethanol (tyrosol, referred to as Tyr) undergoes an extensive first-pass intestinal/hepatic metabolism. ${ }^{3-5}$ As a consequence, the concentration of $\mathrm{Tyr}$ in biological fluids may be extremely low compared to their metabolites, $\underline{6}$ which is suggestive of a potential role of Tyr metabolites in supporting health benefits. Nevertheless, Tyr has been previously shown to inhibit the generation of $\mathrm{H}_{2} \mathrm{O}_{2}$, to reduce lipid peroxidation products in murine J774A.1 and RAW264.7 macrophages, ${ }^{7}$ the phosphorylation of $\mathrm{NF}-\kappa \mathrm{B}$ in human U-87 glioblastoma cells, $\stackrel{8}{\text { the protein }}$ expression of ICAM-1 and monocyte adhesion in human endothelial hybrid EA.hy.926 cells, ${ }^{\underline{9}}$ and to protect myocardium from ischemia-related stress in rats. ${ }^{10}$ However, Tyr was ineffective to influence VCAM-1 gene expression ${ }^{\underline{11}}$ or secretion ${ }^{\underline{12}}$ in human umbilical vein endothelial cells.

The known three main metabolic modifications of Tyr are glucuronation, sulphation, and methylation from which 4'-O-D- $\beta$-glucuronate (referred to as Tyr-GLU) and 4-Osulphate (referred to as Tyr-SUL) are the most abundant metabolites in human plasma (Fig. 1) ${ }^{3,5}$ In a recent study, we reported that Tyr-SUL protects human enterocyte-like cells against the pro-oxidant effects of oxidized cholesterol. $\frac{13}{3}$ The data from human trials have also revealed that Tyr-SUL may be, at least in part, carried by plasma LDL after the ingestion of virgin olive oil. ${ }^{3,14}$ Thus, phenols that bind to LDL could exert their antioxidant action in the arterial intima where the LDL oxidation occurs. The endothelium is involved in the early events of atherosclerosis. ${ }^{15,16}$ Vascular endothelial cells function as a docking site for circulating leukocytes when oxidative and inflammatory pathways become activated by internal or external stimuli such as tumor necrosis factor alpha (TNF- $\alpha$ ), oxidized LDL, or LPS. $\frac{15-17}{}$ Such stimuli, particularly TNF- $\alpha$ in different models of inflammation, $\frac{18,19}{1}$ disturb the reduction-oxidation (redox) balance, and lead to a pro-inflammatory state of endothelial cells in a process mediated by NF- $\kappa$ B. Since the biological properties of Tyr metabolites compared with Tyr on human umbilical vein endothelial cells (hECs) are still unknown, this study was aimed to evaluate the antioxidant and anti-inflammatory effects of Tyr and chemically synthesized Tyr-GLU and Tyr-SUL metabolites in TNF- $\alpha$-treated hECs. In addition, the anti-inflammatory effects in vivo were assessed in mice treated with carrageenan and 12-O-tetradecanoylphorbol-13-acetate (TPA) as models of acute and chronic inflammation. 


\section{Materials and methods}

\section{Synthesis of Tyr metabolites}

Tyr was obtained from Sigma-Aldrich (Madrid, Spain). Immobilized lipase Novozym 435 ${ }^{\circledR}$ was a gift from Novozymes (Madrid, Spain). All other chemicals obtained from commercial sources were used without further purification, unless otherwise noted. All reactions were monitored by thin-layer chromatography (TLC) on plates pre-coated with silica gel 60 F254 and detected by heating with 5\% sulphuric acid in ethanol or Mostain [500 mL of $10 \% \mathrm{H}_{2} \mathrm{SO}_{4}, 25 \mathrm{~g}$ of $\left(\mathrm{NH}_{4}\right)_{6} \mathrm{Mo}_{7} \mathrm{O}_{24} \cdot 4 \mathrm{H}_{2} \mathrm{O}$, and $1 \mathrm{~g}$ of $\left.\mathrm{Ce}\left(\mathrm{SO}_{4}\right)_{2} \cdot 4 \mathrm{H}_{2} \mathrm{O}\right]$. The products were purified by flash chromatography with Merck silica gel 60 (200-400 mesh). Metabolites were purified by chromatography with Reverse Phase (RP)-C18 silica gel. Low-resolution mass spectra were obtained on an ESI/ion trap mass spectrometer. High-resolution mass spectra were obtained on an ESI/quadrupole AutoSpec-Q mass spectrometer. Nuclear magnetic resonance (NMR) spectra were recorded on a 300, 400 or $500 \mathrm{MHz}$ [300 or $400 \mathrm{MHz}\left({ }^{1} \mathrm{H}\right), 75$ or 100 $\left({ }^{13} \mathrm{C}\right)$ ] spectrometer, at room temperature for solutions in $\mathrm{CDCl}_{3}, \mathrm{D}_{2} \mathrm{O}$ or $\mathrm{CD}_{3} \mathrm{OD}$. 2D NMR experiments (COSY, TOCSY, ROESY, and HMQC) were carried out when necessary to assign the corresponding signals of the new compounds. Samples were lyophilized to dryness three times from $\mathrm{D}_{2} \mathrm{O}$ to deuterate all exchangeable protons. Raw data were multiplied by using a shifted exponential window function prior to Fourier transformation, and the baseline was corrected using polynomial fitting. For details on the synthesis of Tyr-GLU and Tyr-SUL metabolites, see ESI Materials and methods. I

\section{Cell cultures}

hECs (human umbilical vein endothelial cells) were obtained from Lonza (CC2517A; Basel, Switzerland) and grown in EBM-2 medium (Lonza, CC-3156) supplemented with the SingleQuot Kit (Lonza, CC-4176) up to the fifth passage. The human monocytic cell line THP-1 was obtained from the American Type Culture Collection (TIB-202; Rockville, MD, USA) and grown in RPMI-1640 medium containing 10\% FBS, $2 \mathrm{mM}$ glutamine, $100 \mathrm{U}$ per $\mathrm{mL}$ penicillin, and $0.1 \mathrm{mg}$ per $\mathrm{mL}$ streptomycin. The cells were checked for possible mycoplasma contamination using the fluorescent dye 4,6-diamidino-2-phenylindole (DAPI) (Sigma-Aldrich) and examined under a motorized inverted fluorescence microscope IX81 equipped with a 100× objective and a Megaview-II digital camera (Olympus, Tokyo, Japan).

\section{Cell viability assay}

hECs were cultured in 96-well plates in eight replicate sets at a density of $10^{4}$ cells per well in the presence of Tyr or its metabolites at the indicated concentrations for $48 \mathrm{~h}$. The cell viability was assayed based on the ability of live cells to reduce methylthiazolyldiphenyl-tetrazolium bromide (MTT). $\stackrel{20}{ }$

\section{ROS analysis}

The intracellular ROS was determined using the CellROX Green Reagent (ThermoFisher Scientific, Madrid, Spain). hECs were exposed to Tyr or its metabolites $(100 \mu \mathrm{M})$ for $16 \mathrm{~h}$. Thereafter, the cells were treated with TNF- $\alpha$ (10 ng $\mathrm{mL}^{-1}$; 
Peprotech, Rocky Hill, NJ, USA) for $1 \mathrm{~h}$ and then with CellROX Green Reagent (5 $\mu \mathrm{M})$ for $30 \mathrm{~min}$. The cells were washed with PBS, fixed with 3.7\% formaldehyde, and the fluorescence signal was analysed in a Fluoroskan Microplate Fluorometer (ThermoFisher Scientific) equipped with a 485/555 excitation/emission filter set. The auto-fluorescence of the cells was measured under the same conditions but without adding CellROX Green Reagent.

\section{GSH assay}

hECs were exposed to Tyr or its metabolites $(100 \mu \mathrm{M})$ for $16 \mathrm{~h}$. Thereafter, the cells were treated with TNF- $\alpha\left(10 \mathrm{ng} \mathrm{mL}{ }^{-1}\right)$ for $24 \mathrm{~h}$. Cell extracts were obtained in 5\% sulphosalicylic acid followed by two freeze/thaw cycles. ${ }^{21}$ GSH was determined in samples of cell extracts by measuring the formation of p-nitrophenol from 5,5'dithiobis(2-nitrobenzoic acid) in the presence of GSH reductase and the reduced form of nicotinamide adenine dinucleotide phosphate using the GSH Assay Kit (CS0260; Sigma-Aldrich).

\section{Immunoblotting}

hECs were exposed to Tyr or its metabolites $(100 \mu \mathrm{M})$ for $16 \mathrm{~h}$. Thereafter, the cells were treated with TNF- $\alpha\left(10 \mathrm{ng} \mathrm{mL}{ }^{-1}\right)$ for $6 \mathrm{~h}$. Total cell proteins, extracted from hECs under different experimental conditions, were examined by western blot analysis as

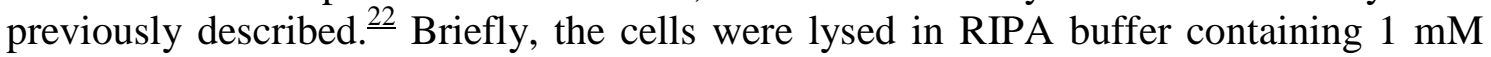
$\mathrm{Na}_{3} \mathrm{VO}_{4}, 1 \mathrm{mM} \mathrm{NaF}, 1 \mu \mathrm{g}$ per $\mathrm{mL}$ aprotinin, $1 \mu \mathrm{g}$ per mL leupeptin, $1 \mu \mathrm{g}$ per $\mathrm{mL}$ pepstatin, and $1 \mu \mathrm{M}$ PMSF. Cell homogenates were then centrifuged at $4{ }^{\circ} \mathrm{C}$. Total proteins in the supernatant were collected, their concentration was determined using the Bradford protein assay, and stored at $-80{ }^{\circ} \mathrm{C}$. Samples were subjected to SDS-PAGE and transferred onto a nitrocellulose membrane $(0.22 \mu \mathrm{m}$, Bio-Rad Laboratories). The protein loading was confirmed by reversible Ponceau S staining. The membranes were immunoblotted with goat anti-HO-1 (C-18, sc-1796; Santa Cruz Biotechnology, Santa Cruz, CA, USA), mouse anti-ICAM-1 (15.2, sc-107), mouse anti-VCAM-1 (E-10, sc13160), and rabbit anti-E-selectin (H-300, sc-14011) antibodies. The main proteins

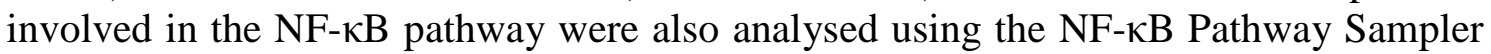
Kit (9936S; Cell Signaling Technology, MA, USA). Specific antigen-antibody complexes were detected using the SuperSignal West Pico Chemiluminescent Substrate (ThermoFisher Scientific). The protein loading equivalence was corrected in relation to the expression of $\beta$-tubulin (T4026; Sigma-Aldrich).

\section{Secretion analysis}

hECs were exposed to Tyr or its metabolites $(100 \mu \mathrm{M})$ for $16 \mathrm{~h}$. Thereafter, the cells were treated with TNF- $\alpha\left(10 \mathrm{ng} \mathrm{mL} \mathrm{mL}^{-1}\right)$ for $16 \mathrm{~h}$. The concentrations of ICAM-1, VCAM-1, and E-selectin in culture supernatants were determined by using commercial ELISA kits (Diaclone, Besancon, France). The values were expressed as $\mathrm{pg} \mathrm{mL}^{-1}$ and calculated from standard curves for each test. DO was measured at $450 \mathrm{~nm}$ on a Multiskan Spectrum plate reader (ThermoFisher Scientific).

\section{RNA isolation and real-time quantitative PCR analysis}


hECs were exposed to Tyr or its metabolites $(100 \mu \mathrm{M})$ for $16 \mathrm{~h}$. Thereafter, the cells were treated with TNF- $\alpha$ (10 ng $\left.\mathrm{mL}^{-1}\right)$ for $3 \mathrm{~h}$. The mRNA levels for specific genes were determined by real-time quantitative PCR using a MX3000P system (Stratagene, La Jolla, CA, USA). Total RNA was extracted from cells with TRIsureTM Reagent (Bioline GmbH, Berlin, Germany). RNA quality was assessed using the OD260 to OD280 ratio, as measured using a NanoDrop ND-1000 Spectrophotometer (ThermoFisher Scientific). Reverse transcription was performed using $1 \mu \mathrm{g}$ of RNA and an iScript Reverse Transcription Kit (Bio-Rad Laboratories, Madrid, Spain). The cDNA template was added to Brilliant SYBR green QPCR Master Mix (Agilent Technologies, Santa Clara, CA, USA) containing the primer pairs for glutathione peroxidase 1 (GPX1), glutamate-cysteine ligase catalytic subunit (GCLC), heme oxygenase-1 (HO1), intercellular adhesion molecule-1 (ICAM-1), vascular adhesion molecule-1 (VCAM1), E-selectin, chemokine ( $\mathrm{C}-\mathrm{C}$ ) motif ligand 2 (CCL2), prostaglandin-endoperoxidase synthase 2 (PTGS2) or reference genes glyceraldehyde-3-phosphate dehydrogenase (GAPDH) and hypoxanthine-guanine phosphoribosyltransferase (HPRT). The sequence and information of the primers used in this study are presented in ESI Table 1. I The reactions were performed in triplicate, and the change in mRNA expression was calculated using the $2^{-(\Delta \Delta C t)}$ method. All data were normalized to the endogenous reference (GAPDH and HPRT) gene levels and expressed as the fold change with respect to the effects of TNF- $\alpha$.

\section{Adhesion assay}

THP-1 monocytes were labelled with $5 \mu \mathrm{M}$ calcein-AM (C3100MP; Molecular Probes, Oregon, USA) for $30 \mathrm{~min}$ and then seeded $(2.5 \times 105$ cells) over hECs previously exposed to Tyr or its metabolites $(100 \mu \mathrm{M})$ for $16 \mathrm{~h}$ and to TNF- $\alpha\left(10 \mathrm{ng} \mathrm{mL} \mathrm{mL}^{-1}\right)$ for additional $6 \mathrm{~h}$. After the co-culture, the cells were washed with PBS and fluorescence was measured at excitation and emission wavelengths of $485 \mathrm{~nm}$ and $530 \mathrm{~nm}$, respectively, using a Fluoroskan Microplate Fluorometer (ThermoFisher Scientific). THP-1 cells adhered to hECs were visualized by fluorescence microscopy with a motorized inverted fluorescence microscope IX81 equipped with a FITC filter and a Megaview-II digital camera (Olympus).

\section{Animals}

Forty-eight male Swiss albino mice (Mus musculus) aged 5 weeks with a body weight of 20-25 g were used for the present study. The animals were maintained under controlled temperature and light conditions in an animal house, and were provided standard mice feed and water ad libitum. Mice were divided into 8 groups with each group containing 5 mice. The dose of Tyr was chosen based on previous studies. ${ }^{23}$ All animal care and experimental procedures complied with the Guidelines of the European Union regarding animal experimentation (Directive of the European Counsel 86/609/EC) and followed a protocol observed and approved by the Animal Ethics Committee of the University of Seville.

\section{Carrageenan-induced hind paw oedema}

Tyr (0.5 mg kg-1) or Tyr-SUL ( 0.1 and $\left.0.5 \mathrm{mg} \mathrm{kg}^{-1}\right)$ was intraperitoneally injected to animals 30 min before the induction of oedema with carrageenan. Oedema was induced by injection of $0.1 \mathrm{~mL}$ of a freshly prepared $1 \%(\mathrm{w} / \mathrm{v})$ carrageenan in sterile saline 
solution $(0.9 \% \mathrm{NaCl})$ into the right hind foot of each mouse under the subplantar aponeurosis. ${ }^{24}$ The control group received sterile saline solution with no carrageenan. The paw volume was measured in $\mathrm{mL}$ using a plethysmometer (LE7500; Leticia, Madrid, Spain) before carrageenan injection $\left(\mathrm{V}_{0}\right)$ and $1,2,3$, and 5 h post-carrageenan injections $\left(\mathrm{V}_{\mathrm{t}}\right)$. The increase in volume was taken as the volume of oedema and was calculated as $\mathrm{V}_{\mathrm{t}}-\mathrm{V}_{0}$. The area-under-the-curve (AUC) for each experimental condition from 0 to $5 \mathrm{~h}$ was calculated by the trapezoidal method.

\section{TPA-induced ear oedema}

Mice were anesthetized with an intraperitoneal injection of sodium pentothal (31.5 mg $\mathrm{kg}^{-1}$; Braun, Madrid, Spain). Oedema was then induced by topical application of $20 \mu \mathrm{L}$ ( $2.5 \mu \mathrm{g}$ TPA per ear) dissolved in acetone to both surfaces of the right ear of each mouse. ${ }^{25}$ The left ears received the same volume of acetone and were maintained as respective controls. Tyr $\left(0.5 \mathrm{mg} \mathrm{kg}^{-1}\right)$ or Tyr-SUL $\left(0.1\right.$ and $\left.0.5 \mathrm{mg} \mathrm{kg}^{-1}\right)$ was topically applied to animals immediately after TPA application. Inflammation was allowed to develop for $24 \mathrm{~h}$, after which the animals were euthanized by cervical dislocation, and disk sections (6 mm diameter) of the central portion of both ears were obtained and weighed. The oedema, which represented inflammation, was defined as the difference in weight between the disks from the right (treated) and left (negative control) ears.

\section{MPO activity assay}

Ear tissue samples (disks) were homogenized in PBS ( $\mathrm{pH}$ 6.0) containing 0.5\% hexadecyltrimethylammonium bromide and were centrifuged at $13000 \mathrm{~g}$ for $30 \mathrm{~min}$ at 4 ${ }^{\circ} \mathrm{C} .{ }^{23}$ MPO activity was measured in collected supernatants according to the method of Bradley et al. ${ }^{26}$ Enzyme activity was determined by measuring OD at $450 \mathrm{~nm}$. Activity is expressed as OD per biopsy.

\section{Statistical analysis}

The data are presented as the mean \pm SD. The homogeneity of variance was tested using Bartlett's test. For in vitro data, group statistical comparisons were performed by a 1- or 2-way ANOVA with a post-hoc Tukey or Bonferroni test when appropriate. For in vivo data, group statistical comparisons were performed by performing a Kruskal-Wallis test along with a Dunns post-hoc test or a 2-way ANOVA along with a Bonferroni post-hoc test when appropriate. A value of $p<0.05$ was considered statistically significant.

\section{Results}

\section{Synthesis of Tyr metabolites}

Glycosylation of acetyl protected Tyr acceptor $\mathbf{4}$ involving the acetyl protected trichloroacetimidate glucuronosyl donor $\mathbf{5}$ was performed in dry dichloromethane and using $\mathrm{BF}_{3} \cdot \mathrm{OEt}_{2}$ as the promoter (Fig. 2A). The corresponding protected 2-[4'-(methyl2,3,4-tri-O-acetyl- $\beta$-D-glucopyranosyluronate)phenyl] EtOAc was de-acetylated under basic hydrolysis $\left(\mathrm{Na}_{2} \mathrm{CO}_{3}, \mathrm{MeOH}, \mathrm{H}_{2} \mathrm{O}\right)$, and Tyr-GLU 2 could be isolated in high yield $(94 \%)$. 
Tyr-SUL 3 has been synthesized using a protection-deprotection strategy together with the use of microwaves in the critical sulfation step. The primary alcohol was acylprotected using immobilized Candida antarctica lipase Novozym 435 ${ }^{\circledR}$ and vinyl butyrate in tert-butyl methyl ether to afford Tyr butyrate 6 (98\% yield) (Fig. 2B). Next, microwave-assisted sulfation was performed by treatment with the $\mathrm{SO}_{3} \cdot \mathrm{NMe}_{3}$ complex and sulfating reagent, triethylamine in acetonitrile at $100{ }^{\circ} \mathrm{C}$ (98\% yield). The final deprotection with $\mathrm{K}_{2} \mathrm{CO}_{3}$ in $\mathrm{MeOH}$ afforded this metabolite 3 in good yield (94\%).

\section{Tyr and Tyr metabolites on viability of hECs}

The viability of hECs was tested at different concentrations $(0-200 \mu \mathrm{M})$ of Tyr, TyrGLU, and Tyr-SUL for $48 \mathrm{~h}$ (ESI Table 2†). We observed that above 95\% of hECs were able to survive at Tyr or Tyr metabolite concentrations up to $100 \mu \mathrm{M}$. We selected this concentration for further assays since a similar concentration has been used in previous studies in vitro to explore the biological effects of Tyr and other polyphenols. $\underline{11,27-29}$

\section{Tyr and Tyr sulphate suppress TNF- $\alpha$-induced intracellular genes encoding antioxidant enzymes in hECs}

Tyr and Tyr-SUL but not Tyr-GLU were able to suppress the intracellular production of ROS induced by TNF- $\alpha$ in hECs (Fig. 3A). Accordingly, Tyr and Tyr-SUL reestablished the depleted intracellular levels of GSH (Fig. 3B) and the mRNA levels of the antioxidant GPX1 gene (Fig. 3C). Tyr-SUL also restored the expression of the GCLC gene (Fig. 3D), which encodes the first rate-limiting enzyme of GSH synthesis. It was observed that Tyr and Tyr-SUL induced the gene (Fig. 3E) and protein (Fig. 3F and ESI Fig. 1A \pm ) expression of the stress-responsive HO-1.

\section{Tyr and Tyr metabolites suppress TNF- $\alpha$-induced phosphorylation of NF- $\kappa B$ signalling proteins in hECs}

The effects of Tyr and its metabolites on TNF- $\alpha$-induced NF- $\kappa B$ signalling in hECs were determined by western blot analysis (Fig. 4A). Tyr-SUL, and to a lesser extent Tyr

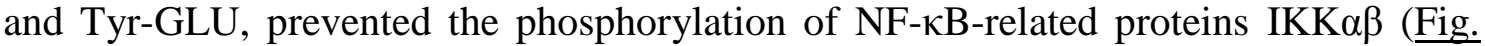
$\underline{\text { 4B and } \mathrm{C}}$ ), I $\kappa \mathrm{B} \alpha$ (Fig. 4D), and p65 (Fig. 4E).

\section{Tyr and Tyr metabolites suppress TNF- $\alpha$-induced up-regulation of adhesion molecules, CCL2, and PTGS2 genes in hECs}

Tyr-GLU and Tyr-SUL, and to a lesser extent Tyr, inhibited the up-regulation of the adhesion molecule gene set induced by TNF- $\alpha$ in hECs such as E-selectin (Fig. 5A) and ICAM-1 (Fig. 5B). Tyr-GLU and Tyr-SUL but not Tyr also suppressed the upregulation of VCAM-1 (Fig. 5C). Comparable effects were found at the protein level (Fig. 5D and ESI Fig. 1B-D士). Accordingly, Tyr metabolites repressed the secretion of soluble E-selectin, ICAM-1, and VCAM-1 while Tyr was only effective in suppressing to a lower extent soluble E-selectin and ICAM-1 (ESI Table 3ذ). The up-regulation of chemoattractant CCL2 (Fig. 5E) and pro-oxidant PTGS2 (Fig. 5F) gene expression was 
also repressed by Tyr-GLU and Tyr-SUL while Tyr only suppressed mRNA levels of the PTGS2 gene but not of the CCL2 gene.

\section{Tyr and Tyr metabolites reduce TNF- $\alpha$-induced adhesion of monocytes to hECs}

Tyr and most notably Tyr-GLU and Tyr-SUL metabolites (Tyr-SUL > Tyr-GLU) reduced the adhesion of human THP-1 monocytes induced by TNF- $\alpha$ in hECs (ESI Fig. 2士).

\section{Tyr and most notably Tyr-SUL reduce the carrageenan-induced paw oedema and TPA-induced ear oedema in mice}

Tyr and Tyr-SUL, as the most active metabolite against the TNF- $\alpha$-induced oxidative and inflammatory response in vitro, were assessed for anti-inflammatory effects in vivo in a mouse model of acute and chronic inflammation using carrageenan and TPA, respectively. The sub-plantar injection of carrageenan caused a noticeable increase in the paw thickness, reaching a peak after $2 \mathrm{~h}$ post-injection (ig. 6A). This effect was reduced when Tyr or Tyr-SUL was injected prior to the treatment with carrageenan. Similar AUC values for paw oedema were obtained after the administration of Tyr at a dose of $0.5 \mathrm{mg} \mathrm{kg}^{-1}$ and Tyr-SUL at a dose of $0.1 \mathrm{mg} \mathrm{kg}^{-1}$ (Fig. 6B). However, Tyr-SUL was more effective than Tyr at the same concentration. As shown in Fig. 6C, TPA caused an increase in the ear disk weight that was reduced after topical application of Tyr and most notably Tyr-SUL. In addition, biopsies from ears treated with Tyr or TyrSUL had reduced the MPO activity (Fig. 6D). This effect was dose-dependent with TyrSUL.

\section{Discussion}

In this study, we have investigated the antioxidant and anti-inflammatory properties relative to those of Tyr of chemically synthesized Tyr-GLU and Tyr-SUL in an in vitro model using TNF- $\alpha$-treated hECs and the anti-inflammatory properties relative to those of Tyr and Tyr-SUL in an in vivo model of acute and chronic inflammation.

In vitro, our data show that Tyr and Tyr-SUL but not Tyr-GLU prevented ROS production, reducing the GSH levels, and GPX1 and GCLC gene transcription induced by TNF- $\alpha$ in hECs, suggesting that glucuronide but not sulphate conjugation of the phenolic hydroxyl group in the Tyr molecule reduces its antioxidant potential. This observation is supported by other studies, in which the antioxidant properties of phenolic compounds such as mangiferin, $\frac{30}{\text { resveratrol, }, \frac{31}{} \text { and quercetin }}{ }^{\frac{32}{}}$ were abolished after hydroxyl conjugation with glucuronic acid in cell-free-systems. GPX1 and GCLC are antioxidant enzymes known to catalyse the reduction of $\mathrm{H}_{2} \mathrm{O}_{2}$ at the expense of two GSH molecules (to create oxidized glutathione, GSSG) and to synthesize new GSH, respectively. $\frac{33,34}{}$ In previous studies, Tyr was shown to attenuate ROS production and to restore the GSH levels and activities of GSH-related enzymes, including GPX, in several murine cell types treated with a mixture of $\mathrm{FeSO}_{4}$ and $\mathrm{H}_{2} \mathrm{O}_{2}, \frac{35}{2} \mathrm{LDL}, \underline{7}$ or subjected to a simulated ischemia/reperfusion process. $\stackrel{29}{ }$ More recently, Tyr inhibited palmitic acid-induced oxidative stress in human liver HepG2 cells and attenuated high 
fat diet-induced lipid peroxidation, restoring the redox equilibrium of GSH in the C57BL/6 mice liver. ${ }^{36}$ The efficiency of Tyr and Tyr-SUL to protect against oxidative stress has been reported in human intestinal Caco-2 cells treated with oxidized LDL ${ }^{37}$ and cholesterol. ${ }^{13}$ HO- 1 is a master cytoprotective gene encoding the enzyme that catalyses the rate-limiting step in the heme catabolism and the breakdown of heme to carbon monoxide, iron, and biliverdin. $\frac{38,39}{}$ In the case of a pro-oxidant and/or proinflammatory stimulus (e.g. TNF- $\alpha$ ), the $5^{\prime}$ untranslated region of the human HO- 1 gene contains antioxidant and stress-activated response elements, including NF- $\kappa B$ sites, ${ }^{40}$ which trigger no less than 200 genes encoding antioxidant, phase-II enzymes, molecular chaperones, and anti-inflammatory and co-stimulatory proteins. ${ }^{41}$ Therefore, our findings on the recovery of TNF- $\alpha$-treated hECs to promote HO-1 gene and protein expression by Tyr and Tyr-SUL could also be indicative of Tyr and Tyr-SUL relevance for the host defence against oxidative stimuli and vulnerability of endothelium to oxidant injury. ${ }^{42}$ TNF- $\alpha$, produced chiefly by activated macrophages, stimulates ROS production and NF- $\mathrm{NB}$ activation, triggering transcription of pro-inflammatory genes in endothelial cells. ${ }^{19}$ Inactive NF- $\kappa$ B is abducted in the cytoplasm by I $\kappa$ B proteins (mainly

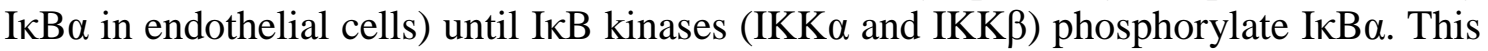
step targets $\mathrm{I} \kappa \mathrm{B} \alpha$ to ubiquitin-mediated proteasomal degradation and phosphorylates NF- $\mathrm{BB}$ (mainly its subunit p65) that is then translocated to the nucleus. $\frac{43}{\text { Tyr }}$ and its metabolites prevented TNF- $\alpha$-induced NF- $\kappa B$ signalling in hECs by reducing the phosphorylation of IKK $\alpha$, IKK $\beta$, I $\kappa \mathrm{B} \alpha$, and p65, with Tyr-SUL being the most effective. These observations are congruent with studies involving Tyr as an inhibitor of NF- $\kappa$ B activation in TNF- $\alpha$-stimulated human glioblastoma cells, ${ }^{-}$IFN $\gamma$ - and LPSstimulated murine macrophages, $, 44,45$ amyloid-beta-stimulated murine neuroblastoma

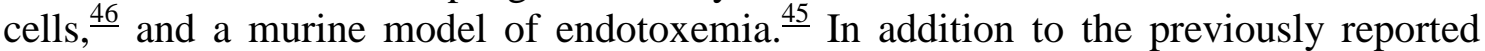
inaction of Tyr on VCAM-1 gene or secretion in hECs,,$\frac{11,12}{2}$ we found that E-selectin, ICAM-1, and VCAM-1 expression and secretion were reduced by both Tyr metabolites, with no differences between them. Other genes involved in the recruitment of monocytes into the arterial wall ${ }^{47}$ and in the pathophysiology of inflammation ${ }^{48}$ such as CCL2 and PTGS2 were similarly down-regulated by Tyr-GLU and Tyr-SUL. It is worth noting the higher potency of Tyr metabolites compared with Tyr and of Tyr-SUL compared with Tyr-GLU in reducing the adhesion of human monocytes to TNF- $\alpha-$ treated hECs. These results altogether support the role of Tyr metabolites, most notably of Tyr-SUL, in the prevention of monocyte trafficking to the blood vessels in inflamed tissues.

Due to the promising results obtained in the in vitro assays, we sought to investigate the effects of Tyr and Tyr-SUL on the course of inflammation in carrageenan-induced paw oedema in mice. We observed that Tyr-SUL was more potent than Tyr in protecting the animals from paw swelling. Tyr-SUL was also particularly effective in reducing the size of ear oedema in TPA-treated mice. In both models, a local inflammation may occur accompanied by the production of ROS, inflammatory cytokines like TNF- $\alpha$, and infiltration of leukocytes. $\stackrel{49}{ }$ While Tyr was previously shown to reduce oedema and MPO activity (an index of the infiltration of leukocytes) in the ears of TPA-treated mice, $\underline{50}$ our study further expand this knowledge and show a higher potency for TyrSUL, in a dose-dependent manner, compared to Tyr. These data suggest that the in vitro antioxidant and anti-inflammatory properties of Tyr-SUL discussed above may play a role in its in vivo anti-oedematogenic capacity. 
The present study has certain strengths and limitations. One strength is that we compared the biological effects of the most important Tyr metabolites with their parent Tyr at equimolar concentrations. Another strength is that we provide evidence for the anti-inflammatory effectiveness of the most prominent active Tyr-SUL from both in vitro and in vivo analyses. A limitation is that the concentration of Tyr, Tyr-GLU, and Tyr-SUL used in vitro is higher than the concentrations described in human plasma after a single ingestion of virgin olive oil. ${ }^{6}$ However, we do not exclude the possibility of Tyr metabolite accumulation in areas of vascular damage, $\stackrel{3,14,51}{~ p a r t i c u l a r l y ~ i n ~ t h e ~ c a s e ~ o f ~}$ Tyr, even Tyr metabolite consumption as nutraceuticals ${ }^{\underline{1}}$ or dietary supplements. $\underline{\underline{52}}$

\section{Conclusions}

In conclusion, this study reveals new biological properties of Tyr metabolites in hECs. We demonstrated that Tyr metabolites ameliorate the TNF- $\alpha$-induced oxidative (TyrSUL $=$ Tyr) and inflammatory (Tyr-SUL $>$ Tyr-GLU $>$ Tyr) status of hECs. We also provide in vivo evidence for Tyr-SUL ameliorating inflammation. Together, these findings reflect the potential of Tyr metabolites, notably Tyr-SUL, as promising antiinflammatory therapeutic agents and offer novel mechanistic explanations underlying the benefits derived from the continued consumption of virgin olive oil or table olives ${ }^{53}$ in the prevention of atherosclerotic disease and other inflammatory-related conditions.

\section{Abbreviations}

AUC Area-under-the-curve

CCL2 Chemokine $(\mathrm{C}-\mathrm{C})$ motif ligand 2

DAPI 4,6-Diamidino-2-phenylindole

GAPDH Glyceraldehyde-3-phosphate dehydrogenase

GCLC Glutamate-cysteine ligase catalytic subunit

GPX1 Glutathione peroxidase 1

GSH Glutathione

hECs Human umbilical vein endothelial cells

HO-1 Heme oxygenase-1

HPRT Hypoxanthine-guanine phosphoribosyltransferase

ICAM-1 Intercellular adhesion molecule 1

MPO Myeloperoxidase

MTT Methylthiazolyldiphenyl-tetrazolium bromide

NF-кB Nuclear factor kappa B

PTGS2 Prostaglandin-endoperoxidase synthase 2

ROS Reactive oxygen species

TNF- $\alpha$ Tumour necrosis factor alpha

TPA 12-O-Tetradecanoylphorbol-13-acetate

Tyr Tyrosol 
Tyr-GLU Tyrosol glucuronate

Tyr-SUL Tyrosol sulphate

VCAM-1 Vascular adhesion molecule 1

\section{Acknowledgements}

We thank Angela Palma Pacheco for technical assistance. This study and the contracts of RL and APP were supported by the research Grant of the Junta de Andalucia, Spain (P09-CVI-5007). SM had the benefit of a FPI fellowship of the MICINN (BES-2012056104) and BB of the "V Own Research Plan" of the University of Seville. SL had Juan de la Cierva contract cofunded by the European Social Fund (ESF) from the Spanish MINECO (JCI-2012-13084,) and the Spanish Research Council/JAEdoc Program (JAEDOC089).

\section{References}

1. S. Rigacci and M. Stefani, Nutraceutical properties of olive oil polyphenols. An itinerary from cultured cells through animal models to humans, Int. J. Mol. Sci., 2016, 17, E483.

2. S. Lopez, B. Bermudez, S. Montserrat-de la Paz, S. Jaramillo, L. M. Varela, A. Ortega-Gomez, R. Abia and F. J. Muriana, Membrane composition and dynamics: a target of bioactive virgin olive oil constituents, Biochim. Biophys. Acta, 2014, 1838, 1638-1656.

3. K. de la Torre-Carbot, J. L. Chavez-Servin, O. Jauregui, A. I. Castellote, R. M. Lamuela-Raventos, M. Fito, M. I. Covas, D. Munoz-Aguayo and M. C. LopezSabater, Presence of virgin olive oil phenolic metabolites in human low density lipoprotein fraction: determination by high-performance liquid chromatographyelectrospray ionization tandem mass spectrometry, Anal. Chim. Acta, 2007, 583, 402-410.

4. R. de la Torre, Bioavailability of olive oil phenolic compounds in humans, Inflammopharmacology, 2008, 16, 245-247.

5. A. Kotronoulas, N. Pizarro, A. Serra, P. Robledo, J. Joglar, L. Rubio, A. Hernaez, C. Tormos, M. J. Motilva, M. Fito, M. I. Covas, R. Sola, M. Farre, G. Saez and R. de la Torre, Dose-dependent metabolic disposition of hydroxytyrosol and formation of mercapturates in rats, Pharmacol. Res., 2013, 77, 47-56.

6. J. Rodriguez-Morato, A. Boronat, A. Kotronoulas, M. Pujadas, A. Pastor, E. Olesti, C. Perez-Mana, O. Khymenets, M. Fito, M. Farre and R. de la Torre, Metabolic disposition and biological significance of simple phenols of dietary origin: hydroxytyrosol and tyrosol, Drug Metab. Rev., 2016, 48, 218-236.

7. R. Di Benedetto, R. Vari, B. Scazzocchio, C. Filesi, C. Santangelo, C. Giovannini, P. Matarrese, M. D'Archivio and R. Masella, Tyrosol, the major extra virgin olive oil compound, restored intracellular antioxidant defences in spite of its weak antioxidative effectiveness, Nutr., Metab. Cardiovasc. Dis., 2007, 17, 535-545.

8. S. Lamy, A. Ben Saad, A. Zgheib and B. Annabi, Olive oil compounds inhibit the paracrine regulation of TNF-alpha-induced endothelial cell migration 
through reduced glioblastoma cell cyclooxygenase-2 expression, J. Nutr. Biochem., 2016, 27, 136-145.

9. C. Manna, D. Napoli, G. Cacciapuoti, M. Porcelli and V. Zappia, Olive oil phenolic compounds inhibit homocysteine-induced endothelial cell adhesion regardless of their different antioxidant activity, J. Agric. Food Chem., 2009, 57, 3478-3482.

10. S. M. Samuel, M. Thirunavukkarasu, S. V. Penumathsa, D. Paul and N. Maulik, Akt/FOXO3a/SIRT1-mediated cardioprotection by n-tyrosol against ischemic stress in rat in vivo model of myocardial infarction: switching gears toward survival and longevity, J. Agric. Food Chem., 2008, 56, 9692-9698.

11. M. A. Carluccio, L. Siculella, M. A. Ancora, M. Massaro, E. Scoditti, C. Storelli, F. Visioli, A. Distante and R. De Caterina, Olive oil and red wine antioxidant polyphenols inhibit endothelial activation: antiatherogenic properties of Mediterranean diet phytochemicals, Arterioscler., Thromb., Vasc. Biol., 2003, 23, 622-629.

12. R. Turner, N. Etienne, M. G. Alonso, S. de Pascual-Teresa, A. M. Minihane, P. D. Weinberg and G. Rimbach, Antioxidant and anti-atherogenic activities of olive oil phenolics, Int. J. Vitam. Nutr. Res., 2005, 75, 61-70.

13. A. Atzeri, R. Lucas, A. Incani, P. Penalver, A. Zafra-Gomez, M. P. Melis, R. Pizzala, J. C. Morales and M. Deiana, Hydroxytyrosol and tyrosol sulfate metabolites protect against the oxidized cholesterol pro-oxidant effect in Caco-2 human enterocyte-like cells, Food Funct., 2016, 7, 337-346.

14. K. de la Torre-Carbot, J. L. Chavez-Servin, O. Jauregui, A. I. Castellote, R. M. Lamuela-Raventos, T. Nurmi, H. E. Poulsen, A. V. Gaddi, J. Kaikkonen, H. F. Zunft, H. Kiesewetter, M. Fito, M. I. Covas and M. C. Lopez-Sabater, Elevated circulating LDL phenol levels in men who consumed virgin rather than refined olive oil are associated with less oxidation of plasma LDL, J. Nutr., 2010, 140, 501-508.

15. P. Libby, Inflammation in atherosclerosis, Nature, 2002, 420, 868-874 .

16. A. Y. Tiong and D. Brieger, Inflammation and coronary artery disease, Am. Heart J., 2005, 150, 11-18.

17. D. Sarmiento, I. Montorfano, M. Caceres, C. Echeverria, R. Fernandez, C. Cabello-Verrugio, O. Cerda, P. Tapia and F. Simon, Endotoxin-induced vascular endothelial cell migration is dependent on TLR4/NF-kappaB pathway, $\mathrm{NAD}(\mathrm{P}) \mathrm{H}$ oxidase activation, and transient receptor potential melastatin 7 calcium channel activity, Int. J. Biochem. Cell Biol., 2014, 55, 11-23.

18. M. Mittal, M. R. Siddiqui, K. Tran, S. P. Reddy and A. B. Malik, Reactive oxygen species in inflammation and tissue injury, Antioxid. Redox Signaling, 2014, 20, 1126-1167.

19. H. Blaser, C. Dostert, T. W. Mak and D. Brenner, TNF and ROS Crosstalk in Inflammation, Trends Cell Biol., 2016, 26, 249-261.

20. S. Jaramillo, S. Lopez, L. M. Varela, R. Rodriguez-Arcos, A. Jimenez, R. Abia, R. Guillen and F. J. Muriana, The flavonol isorhamnetin exhibits cytotoxic effects on human colon cancer cells, J. Agric. Food Chem., 2010, 58, 1086910875.

21. X. Yan, F. Liang, D. Li and J. Zheng, Ouabain elicits human glioblastoma cells apoptosis by generating reactive oxygen species in ERK-p66SHC-dependent pathway, Mol. Cell. Biochem., 2015, 398, 95-104.

22. L. M. Varela, S. Lopez, A. Ortega-Gomez, B. Bermudez, I. Buers, H. Robenek, F. J. Muriana and R. Abia, Postprandial triglyceride-rich lipoproteins regulate 
perilipin-2 and perilipin-3 lipid-droplet-associated proteins in macrophages, J. Nutr. Biochem., 2015, 26, 327-336.

23. S. Silva, B. Sepodes, J. Rocha, R. Direito, A. Fernandes, D. Brites, M. Freitas, E. Fernandes, M. R. Bronze and M. E. Figueira, Protective effects of hydroxytyrosol-supplemented refined olive oil in animal models of acute inflammation and rheumatoid arthritis, J. Nutr. Biochem., 2015, 26, 360-368.

24. A. M. Quilez, S. Montserrat-de la Paz, R. De la Puerta, M. A. Fernandez-Arche and M. D. Garcia-Gimenez, Validation of ethnopharmacological use as antiinflammatory of a decoction from Annona Muricata Leaves, Afr. J. Tradit., Complementary Altern. Med., 2015, 12, 14-20.

25. M. Del-Angel, A. Nieto, T. Ramirez-Apan and G. Delgado, Anti-inflammatory effect of natural and semi-synthetic phthalides, Eur. J. Pharmacol., 2015, 752, $40-48$.

26. P. P. Bradley, R. D. Christensen and G. Rothstein, Cellular and extracellular myeloperoxidase in pyogenic inflammation, Blood, 1982, 60, 618-622.

27. K. Hagiwara, T. Goto, M. Araki, H. Miyazaki and H. Hagiwara, Olive polyphenol hydroxytyrosol prevents bone loss, Eur. J. Pharmacol., 2011, 662, 78-84.

28. E. Scoditti, N. Calabriso, M. Massaro, M. Pellegrino, C. Storelli, G. Martines, R. De Caterina and M. A. Carluccio, Mediterranean diet polyphenols reduce inflammatory angiogenesis through MMP-9 and COX-2 inhibition in human vascular endothelial cells: a potentially protective mechanism in atherosclerotic vascular disease and cancer, Arch. Biochem. Biophys., 2012, 527, 81-89.

29. L. Sun, H. Fan, L. Yang, L. Shi and Y. Liu, Tyrosol prevents ischemia/reperfusion-induced cardiac injury in H9c2 cells: involvement of ROS, Hsp70, JNK and ERK, and apoptosis, Molecules, 2015, 20, 3758-3775.

30. J. D. van der Merwe, E. Joubert, M. Manley, D. de Beer, C. J. Malherbe and W. C. Gelderblom, Mangiferin glucuronidation: important hepatic modulation of antioxidant activity, Food Chem. Toxicol., 2012, 50, 808-815.

31. D. L. Lu, D. J. Ding, W. J. Yan, R. R. Li, F. Dai, Q. Wang, S. S. Yu, Y. Li, X. L. Jin and B. Zhou, Influence of glucuronidation and reduction modifications of resveratrol on its biological activities, ChemBioChem, 2013, 14, 1094-1104.

32. J. G. Messer, R. G. Hopkins and D. E. Kipp, Quercetin metabolites up-regulate the antioxidant response in osteoblasts isolated from fetal rat calvaria, J. Cell. Biochem., 2015, 116, 1857-1866.

33. A. L. Stefanson and M. Bakovic, Dietary regulation of Keap1/Nrf2/ARE pathway: focus on plant-derived compounds and trace minerals, Nutrients, 2014, 6, 3777-3801.

34. Y. Zhang, in Encyclopedia of Cancer, ed. M. Schwab, Springer Berlin Heidelberg, Berlin, Heidelberg, 2012, pp. 2853-2855, DOI:10.1007/978-3-64216483-5_4510.

35. S. Martin, E. Gonzalez-Burgos, M. E. Carretero and M. P. Gomez-Serranillos, Protective effects of Merlot red wine extract and its major polyphenols in PC12 cells under oxidative stress conditions, J. Food Sci., 2013, 78, H112-H118.

36. L. K. Sarna, V. Sid, P. Wang, Y. L. Siow, J. D. House and K. O, Tyrosol attenuates high fat diet-induced hepatic oxidative stress: Potential involvement of cystathionine beta-synthase and cystathionine gamma-lyase, Lipids, 2016, 51, 583-590.

37. C. Giovannini, E. Straface, D. Modesti, E. Coni, A. Cantafora, M. De Vincenzi, W. Malorni and R. Masella, Tyrosol, the major olive oil biophenol, protects 
against oxidized-LDL-induced injury in Caco-2 cells, J. Nutr., 1999, 129, 12691277.

38. L. E. Otterbein, R. Foresti and R. Motterlini, Heme oxygenase-1 and carbon monoxide in the heart: The balancing act between danger signaling and prosurvival, Circ. Res., 2016, 118, 1940-1959.

39. M. Zhang, C. An, Y. Gao, R. K. Leak, J. Chen and F. Zhang, Emerging roles of Nrf2 and phase II antioxidant enzymes in neuroprotection, Prog. Neurobiol., 2013, 100, 30-47.

40. S. A. Rushworth, K. M. Bowles, P. Raninga and D. J. MacEwan, NF-kappaBinhibited acute myeloid leukemia cells are rescued from apoptosis by heme oxygenase-1 induction, Cancer Res., 2010, 70, 2973-2983.

41. B. Chen, Y. Lu, Y. Chen and J. Cheng, The role of Nrf2 in oxidative stressinduced endothelial injuries, J. Endocrinol., 2015, 225, R83-R99.

42. J. L. Lim, M. M. Wilhelmus, H. E. de Vries, B. Drukarch, J. J. Hoozemans and J. van Horssen, Antioxidative defense mechanisms controlled by Nrf2: state-ofthe-art and clinical perspectives in neurodegenerative diseases, Arch. Toxicol., 2014, 88, 1773-1786.

43. N. Parameswaran and S. Patial, Tumor necrosis factor-alpha signaling in macrophages, Crit. Rev. Eukaryotic Gene Expression, 2010, 20, 87-103.

44. D. De Stefano, M. C. Maiuri, V. Simeon, G. Grassia, A. Soscia, M. P. Cinelli and R. Carnuccio, Lycopene, quercetin and tyrosol prevent macrophage activation induced by gliadin and IFN-gamma, Eur. J. Pharmacol., 2007, 566, 192-199.

45. J. Lu, G. Huang, Z. Wang, S. Zhuang, L. Xu, B. Song, Y. Xiong and S. Guan, Tyrosol exhibits negative regulatory effects on LPS response and endotoxemia, Food Chem. Toxicol., 2013, 62, 172-178.

46. C. St-Laurent-Thibault, M. Arseneault, F. Longpre and C. Ramassamy, Tyrosol and hydroxytyrosol, two main components of olive oil, protect N2a cells against amyloid-beta-induced toxicity. Involvement of the NF-kappaB signaling, Curr. Alzheimer Res., 2011, 8, 543-551.

47. T. Gerhardt and K. Ley, Monocyte trafficking across the vessel wall, Cardiovasc. Res., 2015, 107, 321-330.

48. F. Cipollone and M. L. Fazia, Cyclooxygenase-2 inhibition: vascular inflammation and cardiovascular risk, Curr. Atheroscler. Rep., 2006, 8, 245251.

49. H. Sadeghi, V. Zarezade, H. Sadeghi, M. A. Toori, M. J. Barmak, A. Azizi, M. Ghavamizadeh and M. Mostafazadeh, Anti-inflammatory activity of stachys pilifera benth, Iran Red Crescent Me, 2014, 16, e19259.

50. R. de la Puerta, E. Martinez-Dominguez and V. Ruiz-Gutierrez, Effect of minor components of virgin olive oil on topical antiinflammatory assays, Z . Naturforsch., C: Biosci., 2000, 55, 814-819.

51. M. C. Lopez de las Hazas, L. Rubio, A. Kotronoulas, R. de la Torre, R. Sola and M. J. Motilva, Dose effect on the uptake and accumulation of hydroxytyrosol and its metabolites in target tissues in rats, Mol. Nutr. Food Res., 2015, 59, 1395-1399.

52. R. Rodrigo, M. Libuy, F. Feliu and D. Hasson, Polyphenols in disease: from diet to supplements, Curr. Pharm. Biotechnol., 2014, 15, 304-317.

53. I. D'Antuono, A. Garbetta, B. Ciasca, V. Linsalata, F. Minervini, V. M. Lattanzio, A. F. Logrieco and A. Cardinali, Biophenols from table olive cv bella 
di cerignola: Chemical characterization, bioaccessibility, and intestinal absorption, J. Agric. Food Chem., 2016, 64, 5671-5678. 


\section{Figure captions}

Fig. 1 Structure of tyrosol (Tyr) 1, Tyr glucuronate (Tyr-GLU) 2, and Tyr sulphate (Tyr-SUL) 3.

Fig. 2 Synthesis of Tyr-GLU (A) and Tyr-SUL (B).

Fig. 3 Effects of Tyr, Tyr-GLU, and Tyr-SUL on the production of intracellular ROS (A), accumulation of GSH (B), gene expression of GPX1 (C), GCLC (D), and HO-1 (E), and protein expression of HO-1 (F) in TNF- $\alpha$-treated hECs. Values are shown as mean \pm SD of three separate experiments. Means sharing the same letter are not significantly different from each other $(\mathrm{p}<0.05)$.

Fig. 4 Effects of Tyr, Tyr-GLU, and Tyr-SUL on the phosphorylation of NF-кB signalling proteins (A), including quantitative analysis of IKK $\alpha \beta(B, C), \operatorname{I\kappa B} \alpha(D)$, and p65 (E) in TNF- $\alpha$-treated hECs. Values are shown as mean \pm SD of three separate experiments. Means sharing the same letter are not significantly different from each other $(\mathrm{p}<0.05)$.

Fig. 5 Effects of Tyr, Tyr-GLU, and Tyr-SUL on the gene expression of E-selectin (A), ICAM-1 (B), and VCAM-1 (C), protein expression of E-selectin, ICAM-1, and VCAM1 (D), and gene expression of CCL2 (E) and PTGS2 (F) in TNF- $\alpha$-treated hECs. Values are shown as mean \pm SD of three separate experiments. Means sharing the same letter are not significantly different from each other $(\mathrm{p}<0.05)$.

Fig. 6 Effects of Tyr and Tyr-SUL on the carrageenan-induced paw oedema volume in mice (A), including AUC for the paw oedema volume (B), and on the TPA-induced ear oedema weight (C) and MPO activity (D) in mice. Values are shown as mean \pm SD of five animals. Relative to the paw oedema volume: drug effect, time effect, and interaction effect are all $\mathrm{p}<0.05$ (two-way ANOVA). ${ }^{*} \mathrm{p}<0.05$, carrageenan vs. all compounds at indicated times and ${ }^{\#} \mathrm{p}<0.05$, Tyr $\left(0.5 \mathrm{mg} \mathrm{kg}^{-1}\right)$ vs. Tyr-SUL $(0.5 \mathrm{mg}$ $\mathrm{kg}^{-1}$ ) (Bonferroni post-hoc test). Means sharing the same letter are not significantly different from each other $(\mathrm{p}<0.05)$. 
Figure 1
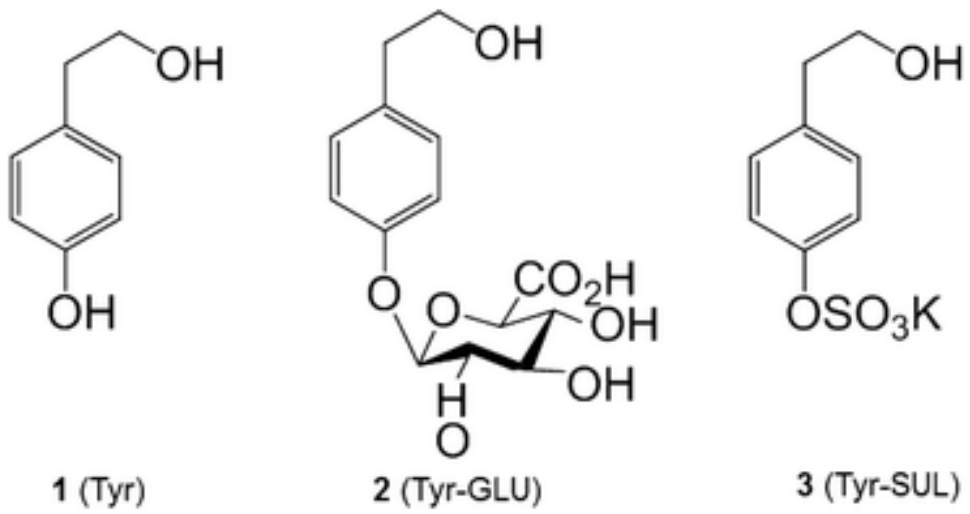
Figure 2

A

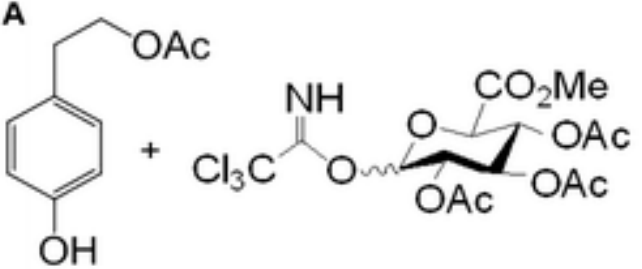

B

4

5<smiles>CCCCOCCc1ccc(O)cc1</smiles>

6

$\mathrm{SO}_{3} \mathrm{NMe}_{3}$

$\mathrm{CH}_{3} \mathrm{CN}$

reaction

$\mid \begin{aligned} & \mathrm{K}_{2} \cdot \mathrm{CO}_{3} \\ & \mathrm{MeOH} \\ & \mathrm{H}_{2} \mathrm{O}\end{aligned}$

CH

$\overbrace{\mathrm{OSO}_{3} \mathrm{~K}}^{\mathrm{OH}}$

2

3 
Figure 3
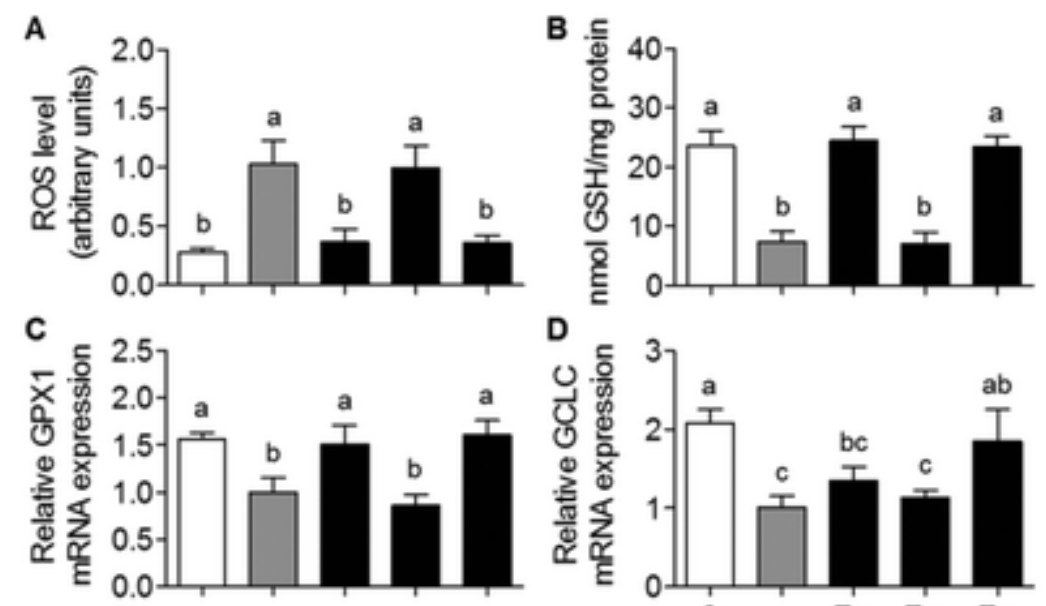

。
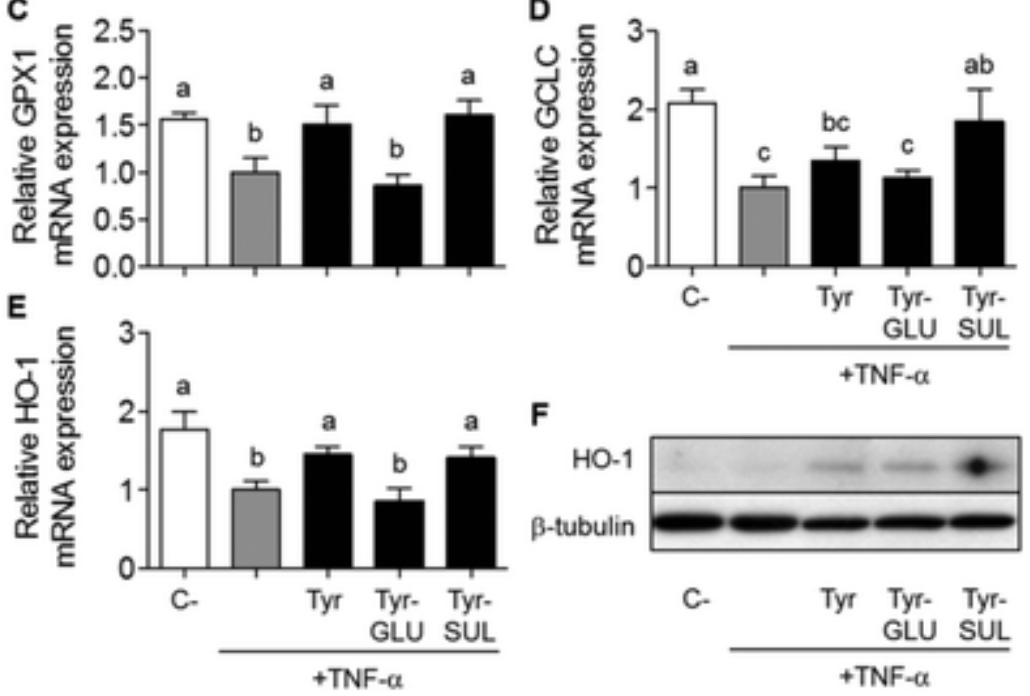
Figure 4

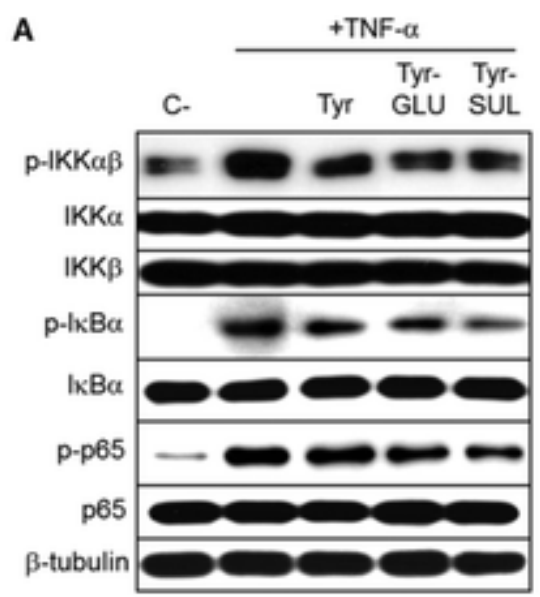

B

C
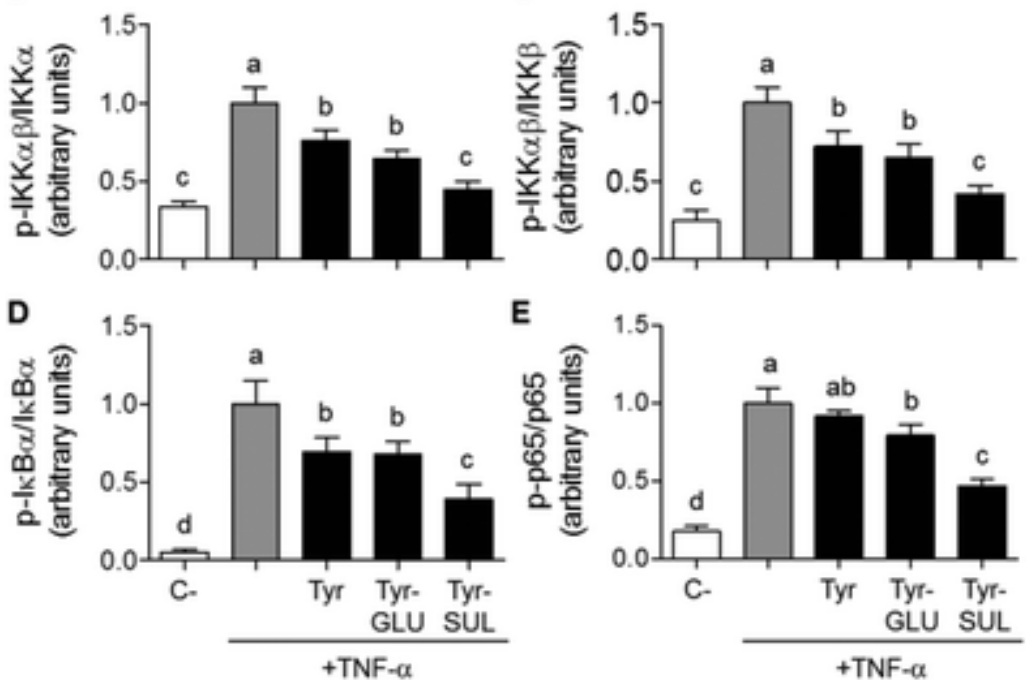
Figure 5

A
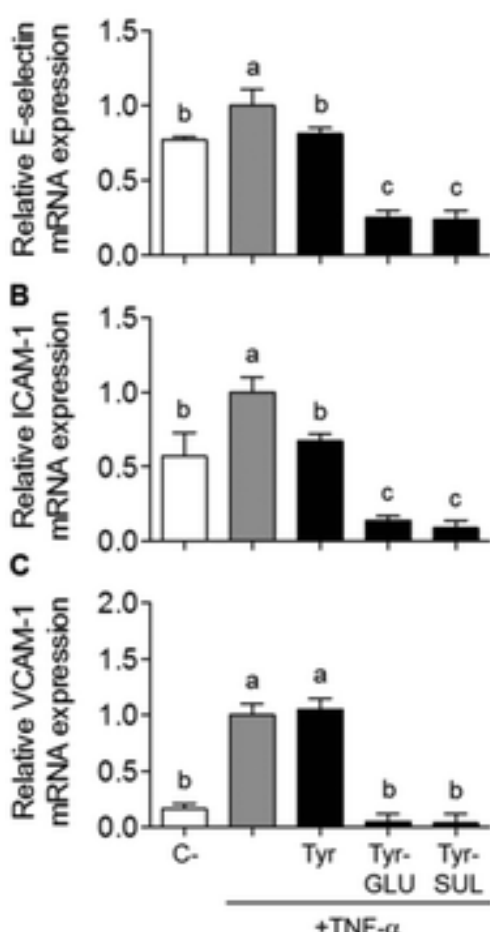

D

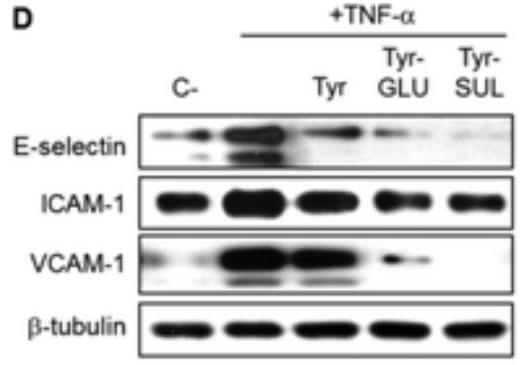

E
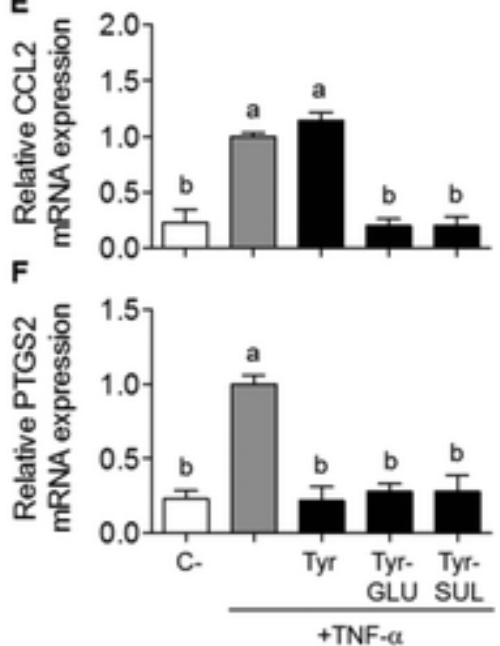
Figure 6

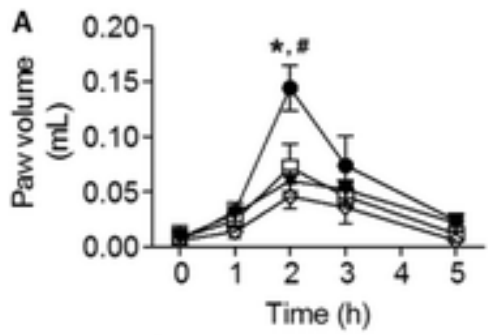

- Carrageenan

* Tyr-SUL (0.1 mg/kg)

$\diamond$ Tyr-SUL $(0.5 \mathrm{mg} / \mathrm{kg}$ )

C

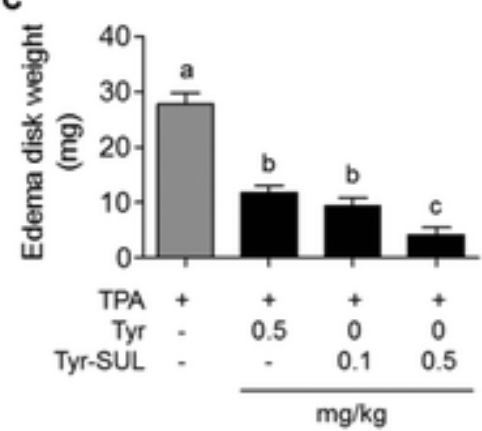

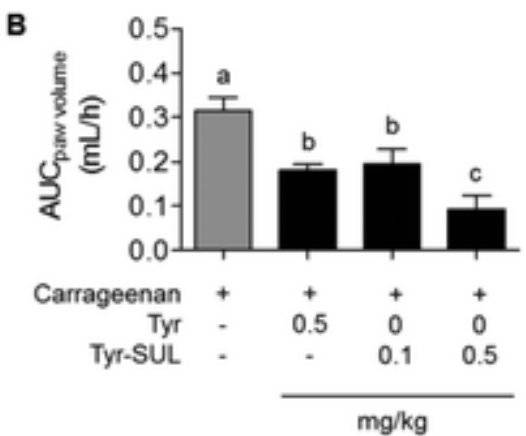

D

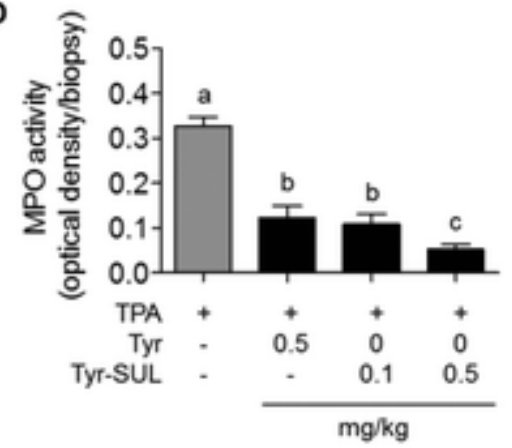

